Schneider-Sliwa R. - Städtische Umwelt im Alter Präferenzen älterer Menschen zum altersgerechten Wohnen, zur Wohnumfeld- und Quartiergestaltung

Basler Stadt- und Regionalforschung, vol. 26, Geographisches Institut der Universität Basel, Bâle, 2004, 150 p.

\title{
Gabriel Wackermann
}

\section{OpenEdition}

\section{Journals}

Édition électronique

URL : http://journals.openedition.org/rge/356

DOI : $10.4000 /$ rge.356

ISSN : 2108-6478

Éditeur

Association des géographes de l'Est

Édition imprimée

Date de publication : 1 juin 2005

Pagination : 221-222

ISSN : 0035-3213

Référence électronique

Gabriel Wackermann, « Schneider-Sliwa R. - Städtische Umwelt im Alter - Präferenzen älterer Menschen zum altersgerechten Wohnen, zur Wohnumfeld- und Quartiergestaltung », Revue Géographique de l'Est [En ligne], vol. 45 / 3-4 | 2005, mis en ligne le 13 mai 2009, consulté le 25 septembre 2020. URL : http://journals.openedition.org/rge/356 ; DOI : https://doi.org/10.4000/rge.356

Ce document a été généré automatiquement le 25 septembre 2020.

Tous droits réservés 


\section{Schneider-Sliwa R. - Städtische Umwelt im Alter - Präferenzen älterer Menschen zum altersgerechten Wohnen, zur Wohnumfeld- und Quartiergestaltung}

Basler Stadt- und Regionalforschung, vol. 26, Geographisches Institut der Universität Basel, Bâle, 2004, 150 p.

\section{Gabriel Wackermann}

\section{RÉFÉRENCE}

Schneider-Sliwa R. - Städtische Umwelt im Alter - Präferenzen älterer Menschen zum altersgerechten Wohnen, zur Wohnumfeld- und Quartiergestaltung, Basler Stadt- und Regionalforschung, vol. 26, Geographisches Institut der Universität Basel, Bâle, 2004, $150 \mathrm{p}$.

1 Financée par une douzaine de sponsors, appuyée sur 11 figures et 11 cartes, en couleur, très expressives, 217 tableaux fort utiles en tant que fondements statistiques d'une méthodologie originale et comme références précises aux diverses étapes de la recherche réalisée par notre collègue Rita Schneider-Sliwa, la présente publication reflète un gros travail d'investigation mené avec l'aide de collaborateurs de l'Institut de géographie de Bâle. La directrice de cet Institut a bénéficié aussi de l'action efficace, en matière de sondage par questionnaire, de l'Association de retraités, par membres interposés de haut niveau. Chaque partie de l'ouvrage est introduite par un assemblage judicieux de photographies en couleur reflétant la thématique traitée.

2 Les annexes, couvrant les pages 117 à 150, comportent, sur 6 pages, le questionnaire de base, très fouillé; elles livrent aussi les données relatives aux recherches 
d'accompagnement relatives à la perception, par les personnes âgées, de la sécurité dans les espaces publics, à l'offre infrastructurelle dans le domaine de l'approvisionnement propre aux retraités, le tout solidement fondé sur des cartes et figures à la fois détaillées et significatives de l'évolution du milieu bâlois. En page 115, l'auteur livre une bibliographie générale, à finalité méthodologique et conceptuelle, qui fait le point sur la littérature plus ou moins marquante parue à ce sujet en espace germanophone. Le contact approfondi avec ces références permet au lecteur de se familiariser à la fois avec les questions qui se rapportent au terrain local proprement dit, et avec les réflexions théoriques alimentées par des ouvrages ou articles de portée générale, suisse et internationale

Rita Schneider-Sliwa comptait initialement, comme tout géographe de pointe, orienter son ouvrage vers une approche interdisciplinaire du thème abordé. Dès l'introduction, elle fait part de son regret de n'avoir pas pu maximiser son investigation. Les partenaires institutionnels et associatifs, directement concernés et intéressés par l'étude, ont toutefois enrichi sensiblement l'approche, centrée sur la recherche des conditions optimales de réussite en matière d'habitation dédiée aux personnes âgées («altersgerechtes Wohnen»). Le questionnaire, ainsi que les enquêtes parallèles et complémentaires, ont bénéficié de ce précieux apport.

4 L'ouvrage est le fruit du questionnement de base suivant: Comment des personnes, principalement âgées de 65 à 85 ans, perçoivent-elles leur environnement résidentiel et, de façon plus générale, leur quartier de vie ? Quels sont leurs aspirations et besoins par rapport à cet environnement? Quel pourrait être le profil de référence le plus adéquat dans le domaine de la planification et de l'aménagement urbains? Quelles sont les mesures de planification à envisager par les collectivités locales et nationales pour conserver aux personnes âgées le plus longtemps possible leur autonomie et reculer sensiblement le moment de la dépendance, posant la question de l'entrée en maison de retraite? Les communautés de personnes âgées, sous toutes leurs formes, depuis les appartements, conçus pour les services à la personne ou une médicalisation plus ou moins prononcée, jusqu'aux maisons de retraite ou de soins, ne sont, en effet, que des recours de dernière heure, non une solution à considérer comme normale, même si le taux de personnes âgées est de plus en plus élevé. Dans le canton de Bâle-ville, par exemple, le taux des plus de 60 ans, par rapport à la population totale, atteint $26 \%$; il est à peu près identique à celui des 20 à 40 ans et à celui des 40 à 60 ans. Le fait est statistiquement établi : dans cette ville, $90 \%$ environ des plus de 80 ans, et $70 \%$ des plus de 90 ans vivent à l'état autonome dans leur propre logement.

Cette situation contribue à expliquer la raison pour laquelle la politique publique des troisième et quatrième âges ne bénéficie pas de réflexions suffisantes sur le devenir des personnes âgées et une meilleure insertion de cette tranche croissante de la population dans les préoccupations urbaines et les projections à plus long terme. D'autant plus que, de surcroît, le grand âge est conçu aussi comme une nouvelle phase de la vie, incitant souvent les personnes concernées à être socialement, culturellement et économiquement actives et utiles pour leurs propres familles et la société dans son ensemble, jusqu'au moment où les forces s'amenuisent, dès lors que ces mêmes personnes avancent dans leur tranche d'âge octogénaire.

6 Après la présentation des motivations de l'étude, de sa signification pour une théorie générale des comportements du troisième âge, et du profil de l'échantillon retenu, Rita Schneider-Sliwa a structuré son ouvrage de la façon suivante, chaque chapitre presque 
étant accompagné d'une synthèse relevant les points essentiels pour un urbanisme adapté aux vœux émis.

7 La seconde partie, subdivisée en six chapitres, est consacrée aux formes résidentielles actuelles et futures des personnes âgées, livrant d'abord les résultats de l'enquête à ce sujet : nature des logements en fonction de la situation familiale, en tant qu'époux ou couple partenarial, état des lieux et recherche d'une adéquation entre les exigences de l'âge et l'offre résidentielle; prise en compte de l'ensemble de la palette des projections personnelles relatives au logement pour personnes âgées, "quatrième » âge inclus; exigences déterminantes pour les résidences destinées aux personnes âgées : sécurité, approvisionnement, services sociaux, qualités environnementales conviviales, infrastructures, qualités résidentielles et de vie; problèmes essentiels soulevés par la question de l'adaptation aux nécessités d'aide à la personne.

8 La troisième partie comporte trois chapitres; elle traite de l'environnement résidentiel et des infrastructures de quartier appelées à desservir des logements pour personnes âgées: perception d'ensemble, potentialités d'approvisionnement, nécessités environnementales ; structures adaptées aux besoins des personnes âgées ; sécurisation des quartiers et des communes concernés.

9 La quatrième partie analyse le profil des deux sexes de troisième âge, au regard de leurs activités et de leur degré de mobilité : champ de relations réel, potentiel souhaitable ; nature des activités, récréatives, touristiques, sportives, domestiques, culturelles et sociales; rayon de mobilité, activités extra-domestiques régulières; facteurs de limitation de la mobilité, choix des moyens de transport; fréquence des déplacements en matière commerciale, conviviale, postale, sanitaire, récréative, religieuse, caritative...

10 La cinquième partie permet de connaître la façon dont les personnes interviewées s'occupent elles-mêmes de leur hébergement, en fonction de l'évolution de leur état général : déménagement dans un rez-de-chaussée, à l'étage, avec ou sans aide à la personne, accessibilité en chaise roulante, orientation vers une maison de retraite ou d'autres formes d'accueil collectif, démarches testamentaires...; nécessité d'une aide lors des courses, des travaux domestiques, des sorties récréatives, aide apportée à d' autres.

11 La sixième partie étudie la manière de vivre le troisième et le quatrième âges: participation au façonnement de l'environnement résidentiel ou du quartier d'habitation ; vœux émis.

12 La publication a le grand mérite de contribuer à faire progresser les recherches fondamentales en gérontologie et à servir de point d'appui à des politiques urbaines destinées à être mieux adaptées aux nécessités et contraintes du monde actuel. En conclusion, l'auteur insiste sur l'importance de la prise de conscience du fait que la société des nouvelles générations de personnes âgées doit être désormais perçue comme un phénomène majeur. Les "vieux » et les associations pour personnes âgées sont également placés face à la nécessité de repenser le rôle des «seniors » des deux sexes dans la société postmoderne. 